\title{
Nanoparticle-mediated RNA interference of angiotensinogen decreases blood pressure and improves myocardial remodeling in spontaneously hypertensive rats
}

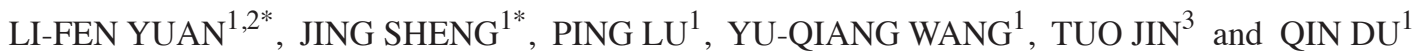 \\ Departments of ${ }^{1}$ Geriatrics and ${ }^{2}$ Respiratory Medicine, Shanghai Ninth People's Hospital, \\ Shanghai Jiao Tong University School of Medicine, Shanghai 200011; \\ ${ }^{3}$ School of Pharmacy, Shanghai Jiao Tong University, Shanghai 200240, P.R. China
}

Received September 9, 2014; Accepted March 23, 2015

DOI: $10.3892 / \mathrm{mmr} .2015 .3909$

\begin{abstract}
Angiotensinogen (AGT) has been shown to have a role in cardiac hypertrophy, while depletion of the AGT gene in spontaneously hypertensive rats (SHR) has not been investigated. The present study investigated the effect of AGT knockdown on cardiac hypertrophy in SHR. For this, small hairpin (sh)RNAs were intravenously injected into SHRs, using a nanoparticle-mediated transfection system. The experimental rats were divided into the following groups: a) Blank control with water treatment only, b) negative control with biscarbamate-crosslinked Gal-polyethylene glycol polyethylenimine nanoparticles (GPE)/negative shRNA, c) AGT-RNA interference (RNAi) group with GPE/AGT-shRNA, and 4) normotensive control using Wistar-Kyoto rats (WKY) with water treatment. Three and five days following the first injection, the levels of hepatic AGT mRNA and AGT protein as well as plasma levels of AGT were markedly decreased in the AGT-RNAi group $(\mathrm{P}<0.05)$. Furthermore, a significant decrease in systolic blood pressure (SBP), left ventricular weight to body weight ratio and heart weight to body weight ratio were observed in the AGT-RNAi group compared with those in the control groups. The depletion of AGT in SHR led to a reduction in SBP by $30 \pm 4 \mathrm{mmHg}$, which was retained for $>10$ days. Cardiac hypertrophy was also significantly improved in AGT-knockdown rats. In conclusion, the present study showed that AGT-silencing had a significant inhibitory
\end{abstract}

Correspondence to: Dr Qin Du, Department of Geriatrics, Shanghai Ninth People's Hospital, Shanghai Jiao Tong University School of Medicine, 639 Zhizaoju Road, Shanghai 200011, P.R. China

E-mail: jeanniedu@hotmail.com

*Contributed equally

Key words: RNA interference, angiotensinogen, angiotensin II, spontaneously hypertensive rats, hypertension effect on hypertension and hypertensive-induced cardiac hypertrophy in SHRs.

\section{Introduction}

Hypertension, a leading risk factor for cardiovascular diseases (1), occurs in $\sim 50 \%$ of aged individuals (2). It has been reported that long-term hypertension is associated with cardiac hypertrophy (3). High blood pressure is associated with the aberrant expression of genes, including angiotensinogen (AGT), which has an important role in the pathogenesis of hypertension (4-6). AGT is secreted by the liver and sequentially cleaved by renin and angiotensin I-converting enzyme $(7,8)$. This results in production of an active hormone, angiotensin II, which induces hypertension (9). AGT has been suggested as an important determinant of blood pressure and cardiac hypertrophy (10). The roles of AGT in the pathogenesis of hypertension have been investigated by molecular genetic approaches, including transgenic animals (11), anti-sense oligodeoxynucleotides (12), linkage analysis of the AGT gene on chromosome 1q42-43 and essential hypertension (13).

Spontaneously hypertensive rats (SHR), have been widely used as an animal model of human primary hypertension $(14,15)$. The mean arterial blood pressure of SHR is significantly increased from 16-20 weeks of age, which is associated with a series of changes in their pathogenesis and pathophysiology (16-18). The pathological changes in SHR are similar to those in humans with primary hypertension $(19,20)$. Therefore, SHR are an ideal animal model for studies on the etiology of human primary hypertension and may serve as a screening model for novel anti-hypertensive drugs (21).

Overexpression of type 1 angiotensin II receptors (AT1R) in renal proximal tubules has been detected in young SHR (22). To explore its role in hypertension, RNA interference (RNAi) techniques have often been used to investigate the functional consequences of AT1R silencing. However, most AT1R-knockdown studies are performed in vitro, while only a few studied have used in vivo animal models $(23,24)$. In the present study, knockdown of AT1R (25) and angiotensin-converting enzyme (ACE) (26) were investigated in SHR, as AT1R and ACE are associated with the function of 
angiotensin II (AngII) in hypertension. Of note, AGT gene expression in SHR has rarely been studied (27).

RNAihas been widely used to selectively inhibit gene expression due to its target specificity. The specific inhibitory effect of small interfering (si)RNA on its target gene is determined by the formation of RNA-induced silencing complexes (28). As RNAi is a useful tool for gene functional studies, it may also potentially be used in gene therapy. However, at present, gene therapy remains challenging due to lack of efficient and safe delivery systems to enable the silencing of a specific gene in the target tissue $(29,30)$. To address this issue, numerous novel gene carriers have been developed, among which cationic polymers are showing promise as a safer strategy for gene delivery, owing to its several advantages, including lower immunogenicity, the ease of chemical modification and the ability to transfer larger plasmid DNA molecules $(31,32)$. Currently, the cationic polymer polyethylenimine (PEI) and its biscarbamate-crosslinked derivatives (PEI-Et) (33) and galactose-polyethylene glycol-PEI-Et (GPE) (34,35), have been successfully used for non-viral transfection with markedly low cytotoxicity, potent transfection efficiency and high hepatocyte-targeting properties. GPE, a hepatocyte-targeting gene carrier, has the ability to condense plasmid DNA (pDNA) into nanoparticles, providing lower cytotoxicity, high transfection efficiency and preferential accumulation in the liver.

In the present study, it was hypothesized that specific RNAi with the AGT gene may have effects on long-term hypertension and may improve hypertensive cardiac hypertrophy. Therefore, effects of AGT-specific small hairpin (sh)RNA coupled to GPE carrier molecules were evaluated in SHRs.

\section{Materials and methods}

Reagents. The GPE carrier was a gift from Professor Jin Tuo of the School of Pharmacy, Jiao Tong University (Shanghai, China). PEI was purchased from Sigma-Aldrich (St. Louis, MO, USA). shRNA plasmids (UUGAUAUCCG) for the negative control (NT) and AGT gene were products of Genechem Biotechnology Co. (Shanghai, China). Plasmid purity was assessed by electrophoresis, and the concentration of pDNA was determined by spectrophotometry (DU-800; Beckman Coulter, Inc., Krefeld, Germany) with absorption at 260/280 nm. The mouse AGT immunoglobulin G (IgG) antibody (cat. no. 77; 1:1,000; overnight incubation at $4^{\circ} \mathrm{C}$ ) was obtained from Swant, Inc. (Belinzona, Switzerland) and the AGT and AngII ELISA Assay kits were purchased from R\&D Systems Europe Ltd. (Abingdon, UK).

Construction of GPE-AGT-shRNA nanoparticles. GPE-AGT-shRNA complexes were prepared by vigorous mixing of GPE solution and plasmid solution of AGT shRNA or negative shRNA at a weight ratio of $30: 1$. The mixture was incubated at room temperature for 1 hour. Since the GPE/DNA weight ratio is a major cytotoxic determinant of the nanoparticles, the optimal GPE/DNA weight ratio (30:1) with the lowest cytotoxicity and the highest transfection efficiency was determined in preliminary experiments. All GPE-AGT-shRNA nanoparticles were freshly prepared prior to the experiments. The nanoparticles were filtered through a 0.2-mm membrane (Millipore Corp., Billerica, MA, USA).
Experimental animals. The present study utilized sixty-one

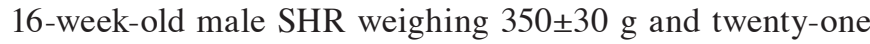
Wistar-Kyoto rats (WKY) with similar features to those of SHR were purchased from the SLAC Laboratory Animal Co., Ltd (Shanghai, China). Rats were housed at three per cage, under a regular 12-hour diurnal light cycle, with free access to food and water during the entire study. All procedures of the animal study were approved by the local ethics committee of the School of Medicine, Shanghai Jiao-Tong University (Shanghai, China) (no. 0708253).

In vivo experiment. Sixty-three SHRs were randomly divided into three groups ( $\mathrm{n}=21$, each group): 1) Blank control injected with $500 \mu \mathrm{l}$ sterile water via the tail vein; 2) negative control with i.v. injection of $500 \mu \mathrm{l}$ of GPE/negative shRNA complex and 3) AGT-RNAi group with i.v. injection of $500 \mu 1$ of GPE-AGT-shRNA. WKY were used as normotensive controls that were only treated with sterile water $(500 \mu \mathrm{l})$ intravenously. All rats received a series of nine injections during the experiment, in which 10 consecutive days were considered a cycle. The injection was administered on the first day of each cycle. Prior to and after injection, SBP (systolic blood pressure) of the caudal artery was measured by the standard tail-cuff method. At days 0, 3, 5, 7 and 10 after injection, the expression levels of AGT mRNA and protein in tissue samples of the liver were determined by reverse transcription quantitative polymerase chain reaction (RT-qPCR) and western blot analyses. Furthermore, serum/plasma levels of AGT and AngII were measured by ELISA. Liver and kidney functions of experimental animals were also examined using the Fully Automatic Biochemical Analyzer (Beckman Coulter, Inc.). Finally, experimental rats were sacrificed by intraperitoneal injection of $50 \mathrm{mg} / \mathrm{kg}$ pentobarbital solution (Sigma-Aldrich). Specimens of heart tissue were taken for pathological examination. Ratios of HW (heart weight)/BW (body weight) and LVW (left ventricle weight)/BW were calculated.

$R T-q P C R$. Total RNA was extracted from tissue samples of the liver using TRIzol reagent (Invitrogen Life Technologies, Shanghai, China). Total RNA (5 $\mu \mathrm{g}$ ) was reversely transcribed into cDNA in a $20-\mu 1$ mixture using Moloney murine leukemia virus Reverse Transcriptase (Cell Bank of Pharmacology and Toxicology Laboratory, New Medicine and Marine Medicine Research Center of Nanjing University of Chinese Medicine, China., according to the manufacturer's instructions. RT-qPCR was performed using the MX 3000P Sequence Detection system (Stratagene, La Jolla, CA, USA) and SYBR Green PCR kit (Applied Biosystems, Life Technologies, Thermo Fisher Scientific, Waltham, MA, USA). The specific primers for specific genes were synthesized by Invitrogen Life Technologies. Primer sequences for AGT were as follows: Forward, 5'-CATCTTCCCTCGCTCTCTG-3' and reverse, 5'-GCCTCTCATCTTCCCTTGG-3' (175 bp). The housekeeping gene, $\beta$-actin, was used as an internal reference for the normalization of the target genes. $\beta$-actin primers were: Forward, 5'-CAAGGTCATCCATGACAACTTTG-3' and reverse, 5'-GTCCACCACCCTGTTGCTGTAG-3' (217 bp). The PCR protocol included a pre-denaturation at $95^{\circ} \mathrm{C}$ for $10 \mathrm{~min}$ and 40 cycles of denaturation at $95^{\circ} \mathrm{C}$ for $30 \mathrm{sec}$, annealing at $60^{\circ} \mathrm{C}$ for $30 \mathrm{sec}$ and extension at $72^{\circ} \mathrm{C}$ for $45 \mathrm{sec}$. 

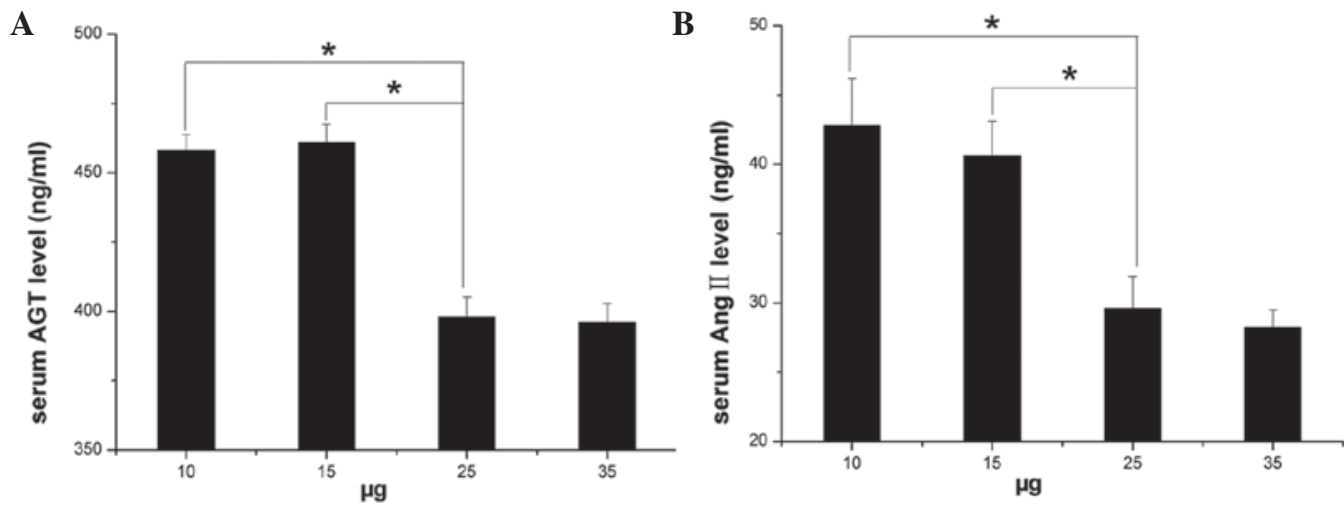

Figure 1. Changes in the serum levels of AGT and AngII in SHR at day 3 after infection with different doese of GPE-AGT-small hairpin RNA nanoparticles. (A) Serum levels of AGT in groups of different doses at day 3 after injection was detected by ELISA. (B) Serum levels of AngII in groups of different doses at day 3 after infection was detected by ELISA. Values are presented as the mean \pm standard error. ${ }^{*} \mathrm{P}<0.05$ as compared with pre-injection levels (day 0). AGT, angiotensinogen; AngII, angiotensin II.

Western blot analysis. $50 \mu \mathrm{g}$ lysates of tissue samples were loaded onto $10 \%$ polyacrylamide gels and separated by SDS-PAGE (Shanghai Biological Technology Co., Ltd., Shanghai, China). The separated proteins were transferred onto nitrocellulose membranes, the membranes were blocked with $0.1 \%$ bovine serum albumin for $2 \mathrm{~h}$ at room temperature and then incubated overnight with mouse anti-rat AGT antibody $(1: 1,000)$ or mouse anti-rat $\beta$-actin antibody $(1: 5,000)$ at $4^{\circ} \mathrm{C}$. After washing three times $(5 \mathrm{~min}$ each) with Tris-buffered saline (10 Mm Tris/ $\mathrm{HCl}, \mathrm{pH} 7.5$ and $150 \mathrm{mM}$ $\mathrm{NaCl}$ ) containing $0.05 \%$ Tween 20 (TBS-T) (Cell Signaling Technology, Inc., Danvers, TX, USA), membranes were incubated with a sheep anti-mouse IgG antibody (cat. no. 0257; 1:1,000; Sigma-Aldrich) for $2 \mathrm{~h}$ at room temperature and then rinsed three times with TBS-T. Finally, blots were developed by enhanced chemiluminescence and exposed to X-ray film (Cell Signaling Technology, Inc.). Quantification of signals was performed by an image acquisition and analysis system (Odyssey v1.2 family of imaging systems; LI-COR Biosciences, Lincoln, NE, USA). Results are presented as the ratio of AGT/B-actin.

Measurement of serum levels of AGT and AngII. Following anesthesia with an intraperitoneal injection of $1 \%$ sodium pentobarbital, blood samples were collected from the femoral vein of the rats. Plasma and cells were then separated by centrifugation for $10 \mathrm{~min}$ at $900 \mathrm{x}$ g at room temperature. The collected plasma was stored at $-80^{\circ} \mathrm{C}$ prior to measurement. Serum levels of AGT and AngII were determined by using ELISA, according to the manufacturer's protocol.

Histological and ultrastructural examination. Sections of heart samples were stained with hematoxylin-eosin (Invitrogen Life Technologies) for the examination of cardiac hypertrophy $(36,37)$. Myocardial ultrastructure was observed under an electron microscope (CMI20; Philips, Amsterdam, Netherlands).

Statistical analysis. All statistical analyses were performed using SPSS software (version 19.0; SPSS, Inc, Chicago, IL). Values are expressed as the mean \pm standard deviation. Student's t-test (two-tailed) was applied to test the significance

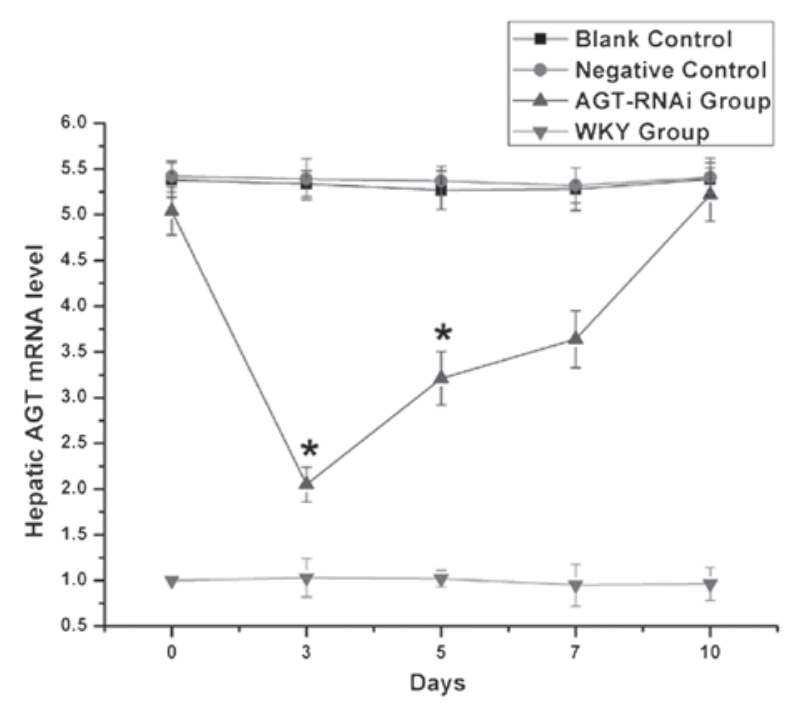

Figure 2. Changes of hepatic mRNA levels of AGT in different groups of SHR prior to and after infection, as determined by reverse transcription polymerase chain reaction. Values are expressed as the mean \pm standard error. ${ }^{*} \mathrm{P}<0.05$ compared between GPE-AGT-small hairpin RNA and the control groups. AGT, angiotensinogen; SHR, spontaneously hypertensive rats; WKY, Wistar-Kyoto rats; RNAi, RNA interference.

of the differences between two groups. $\mathrm{P}<0.05$ was considered to indicate a statistically significant difference between values.

\section{Results}

Dose-dependent effect of injection of GPE-AGT-shRNA nanoparticles. Toinvestigate the efficacy of GPE-AGT-shRNA nanoparticles to inhibit plasma levels of AGT in vivo, different doses of GPE-AGT-shRNA (AGT-shRNA plasmids coupled to GPE carrier molecules, ranging from 10 to $35 \mu \mathrm{g}$ ) were intravenously injected into SHR via the tail vein. As shown in Fig. 1, at day 3 after injection of GPE-AGT-shRNA, the plasma levels of angiotensinogen were significantly decreased $(\mathrm{P}<0.05)$ in a dose-dependent manner, as compared with pre-injection levels (day 0). Results showed a significant inhibitory effect on the plasma levels of angiotensinogen by injection of GPE-AGT-shRNA at doses of 25 


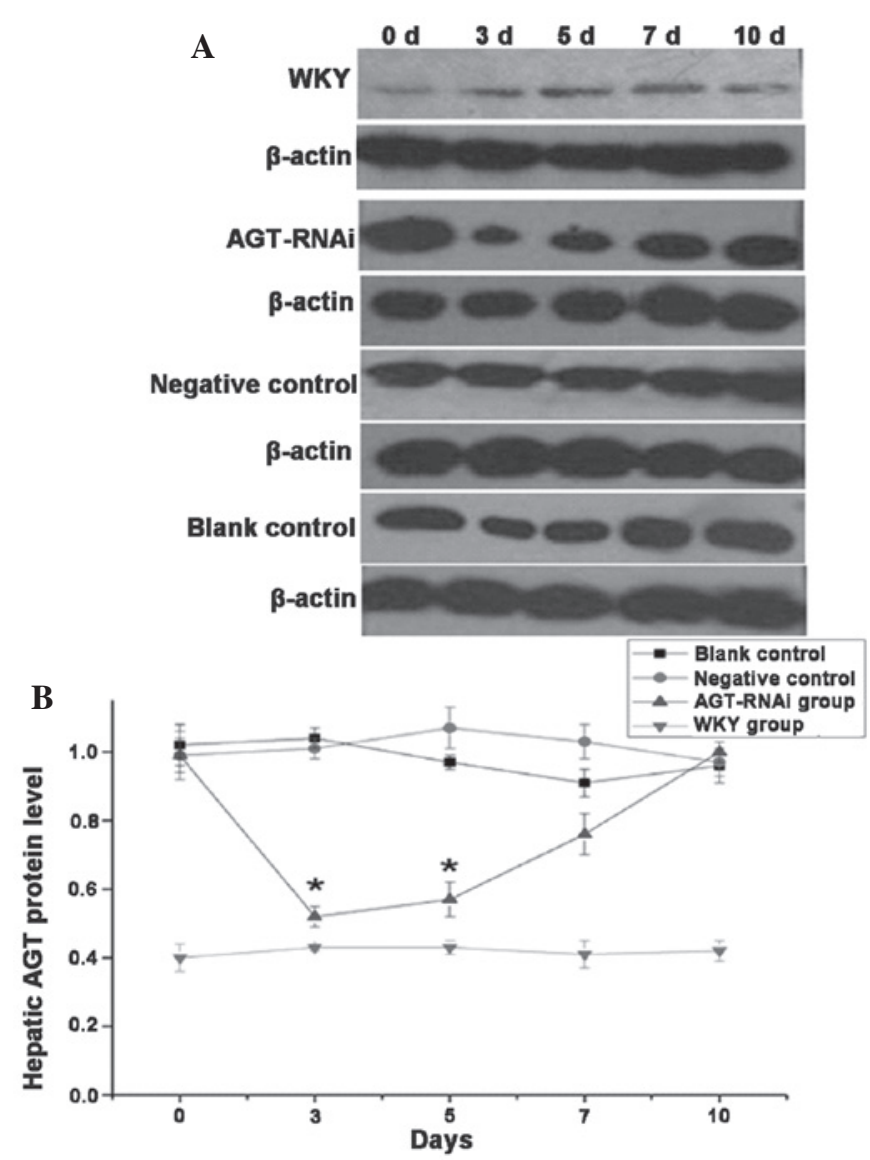

Figure 3. Changes in hepatic protein levels of AGT in different SHR groups, prior to and after infection. (A) Hepatic AGT protein levels in different SHR groups were determined using western blot analysis. $\beta$-actin as the internal control was included in each group. (B) Results of normalized AGT protein from six independent experiments are presented as the mean \pm standard error. " $\mathrm{P}<0.05$ for comparisons between GPE-AGT-small hairpin RNA and the control groups. AGT, angiotensinogen; SHR, spontaneously hypertensive rats; WKY, Wistar-Kyoto rats; RNAi, RNA interference.

and $35 \mu \mathrm{g}$, but no difference was observed between these two doses of GPE-AGT-shRNA $(\mathrm{P}<0.05)$. Therefore, $25 \mu \mathrm{g}$ GPE-AGT-shRNA was selected as an optimal dose for injection in further SHR experiments.

$m R N A$ levels of AGT in experimental rats. As shown in Fig. 2, at days 3 and 5 after injection, hepatic mRNA levels of AGT in the GPE-AGT-shRNA group were significantly lower than those in the other SHR groups $(\mathrm{P}<0.05)$. However, from days 7-10 after injection, hepatic AGT mRNA expression in GPE-AGT-shRNA group gradually increased, eventually equaling the levels in the SHR control groups. However, there was no significant difference in blood pressure between the GPE-AGT-shRNA and control groups during this period.

Protein levels of AGT in experimental rats. As shown in Fig. 3A, at days 3 and 5 after injection, AGT protein levels in the liver of the GPE-AGT-shRNA group were significantly lower than those in the other SHR groups $(\mathrm{P}<0.05)$, but at days 7 and 10 after injection, its levels in the GPE-AGT-shRNA group were equal to those in the other SHR groups. No significant difference in AGT protein expression was found among SHR groups at days 7 and 10
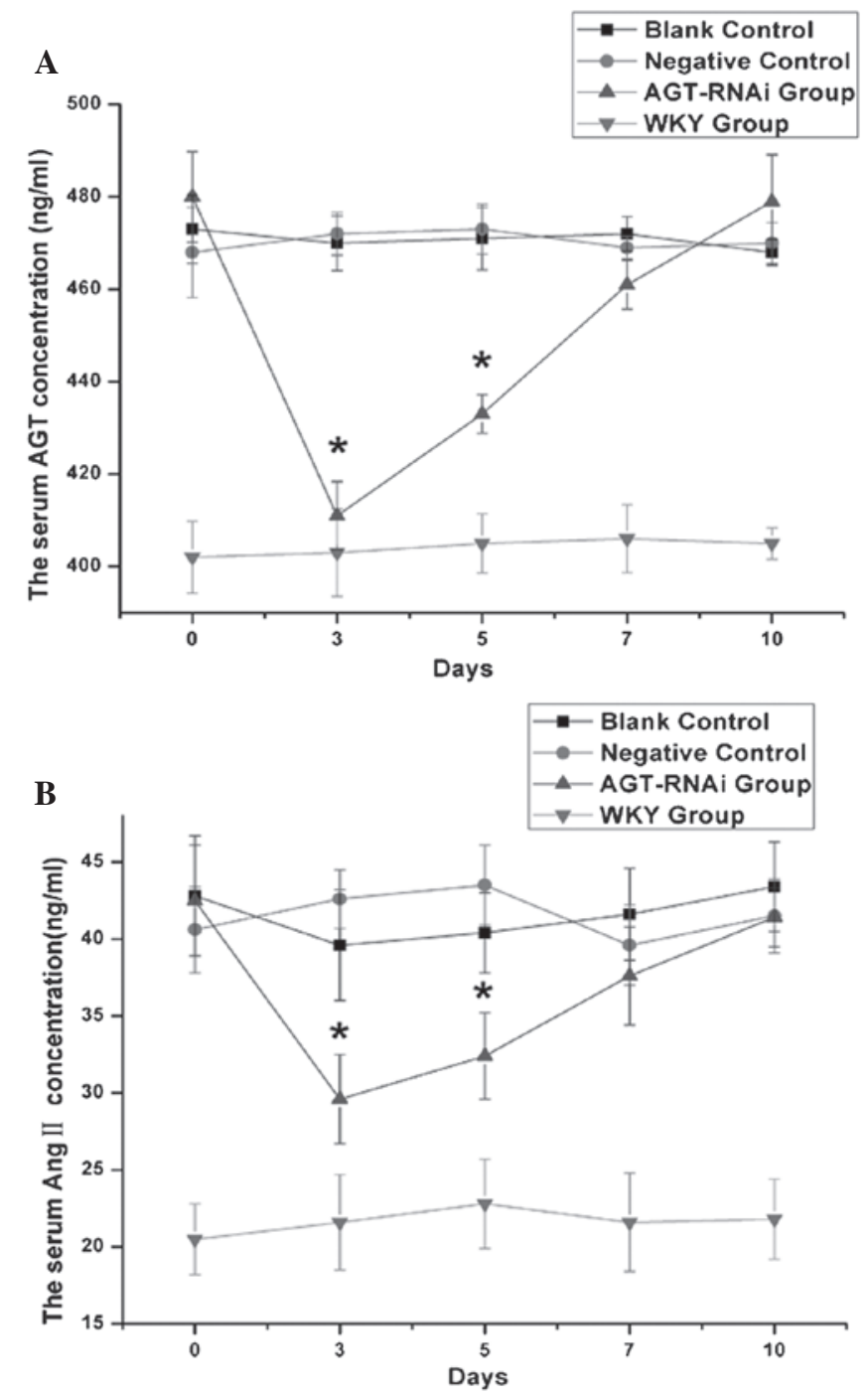

Figure 4. Changes in serum levels of AGT and AngII in different SHR groups, prior to and after infection. (A) Serum levels of AGT in different groups of SHR including WKY control were determined by ELISA. (B) Serum levels of AngII in different groups of SHR including WKY control were determined by ELISA. Values are expressed as the mean \pm standard error. ${ }^{*} \mathrm{P}<0.05$ for comparisons between GPE-AGT-small hairpin RNA and the control groups. AGT, angiotensinogen; SHR, spontaneously hypertensive rats; WKY, Wistar-Kyoto rats; RNAi, RNA interference; AngII, angiotensin II.

after injection. Quantified results of AGT protein expression in the different SHR groups are shown in Fig. 3B.

Serum levels of AGT and AngII in experimental rats. As shown in Fig. 4, at days 3 and 5 after injection, serum levels of AGT (Fig. 4A) and AngII (Fig. 4B) in the GPE-AGT-shRNA group were significantly reduced, and lower than the levels in the other SHR groups $(\mathrm{P}<0.05)$. However, from days 5 to 10 after injection, AGT and AngII levels in the GPE-AGT-shRNA group were gradually increased, eventually equal to those in other SHR groups. No significant differences in serum AGT and AngII levels were observed within the other SHR groups and WKY control at different time points.

Effects of AGT-RNAi on blood pressure. As indicated in Fig. 5, there was a marked decrease in tail arterial pressure in GPE-AGT-shRNA-injected rats, whereas tail arterial 


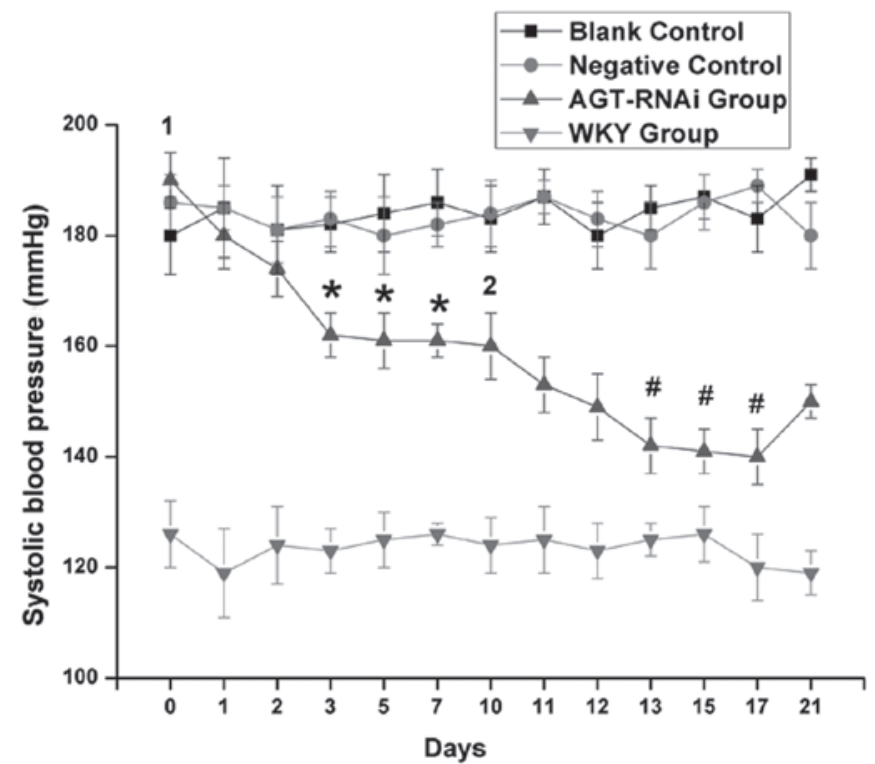

Figure 5. Changes of systolic blood pressure in different groups of SHR prior to and after infection. Tail arterial pressure in different SHR groups was measured daily. Values are expressed as the mean \pm standard error. ${ }^{~} \mathrm{P}<0.05$ for comparisons between GPE-AGT-small hairpin RNA and the control groups. 1, First injection; 2, second injection. AGT, angiotensinogen; SHR, spontaneously hypertensive rats; WKY, Wistar-Kyoto rats; RNAi, RNA interference.

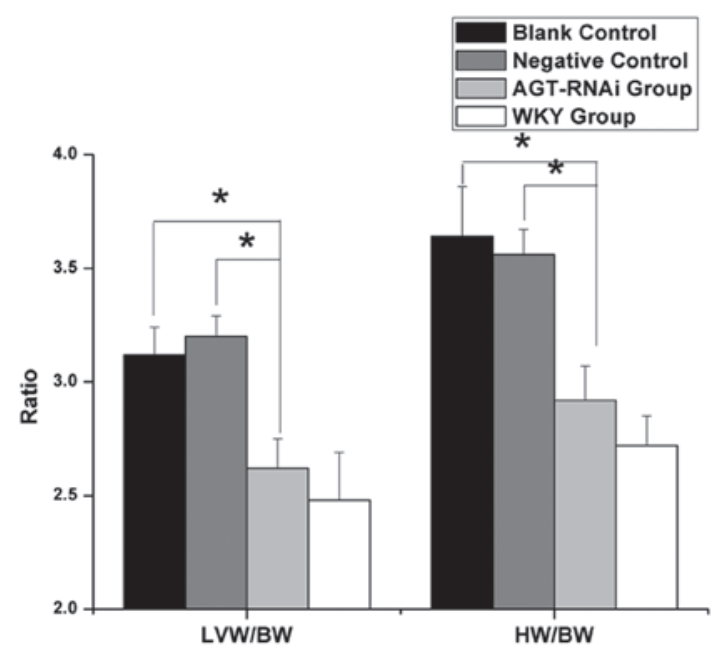

Figure 6. Changes in HW/BW and LVW/BW ratios in experimental rats. At the end of the experiments, the hearts were removed to calculate the $\mathrm{HW} / \mathrm{BW}$ and LVW/BW ratios. Values are expressed as the mean \pm standard error. ${ }^{*} \mathrm{P}<0.05$ for comparisons between GPE-AGT-small hairpin RNA and the control groups. HW, heart weight; BW, body weight; LVW, left ventricle weight; AGT, angiotensinogen; SHR, spontaneously hypertensive rats; WKY, Wistar-Kyoto rats; RNAi, RNA interference.

pressure remained stable in the blank and negative controls. However, no significant changes in BP in the WKY group were observed. The largest decrease in BP was after the 2nd injection accounting up to $53 \mathrm{mmHg}$. The anti-hypertensive effect induced by injection of AGT-shRNA nanoparticles lasted for $>10$ days after each injection.

Changes of $H W / B W$ and $L V W / B W$ in experimental rats. As shown in Fig. 6, the ratios of HW/BW and LVW/BW in
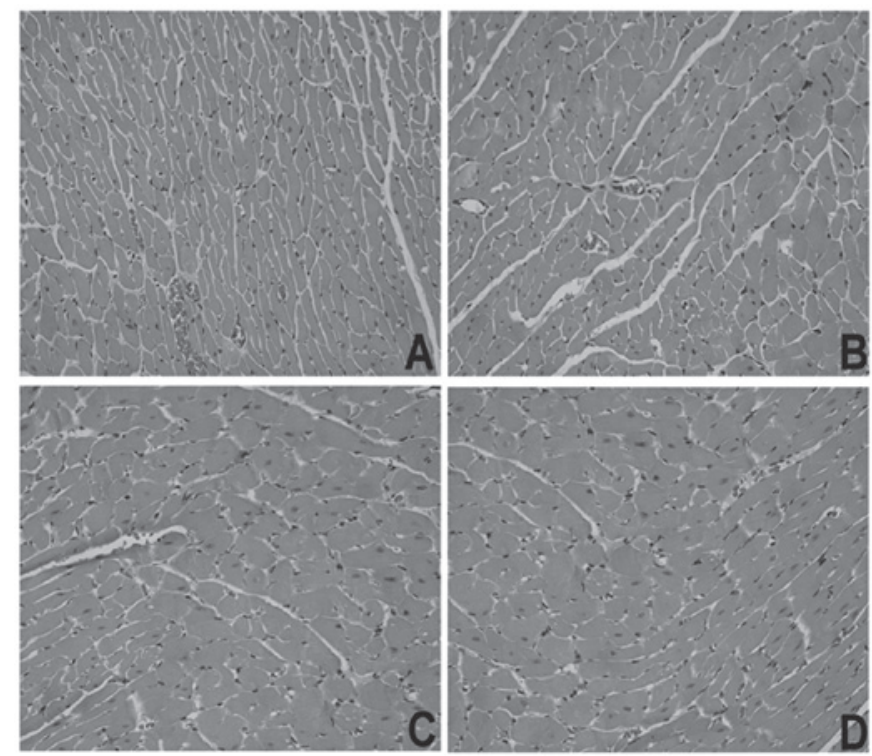

Figure 7. Pathological changes in the myocardial structure. Sections of heart samples from experimental rats were stained with hematoxylin-eosin for cardiac hypertrophy examination. Pathological changes of myocardial structure in each group were examined by optical microscopy (magnification, $\mathrm{x} 200$ ). (A) Wistar-Kyoto rat group; (B) RNA interference group; (C) negative group; (D) blank group.
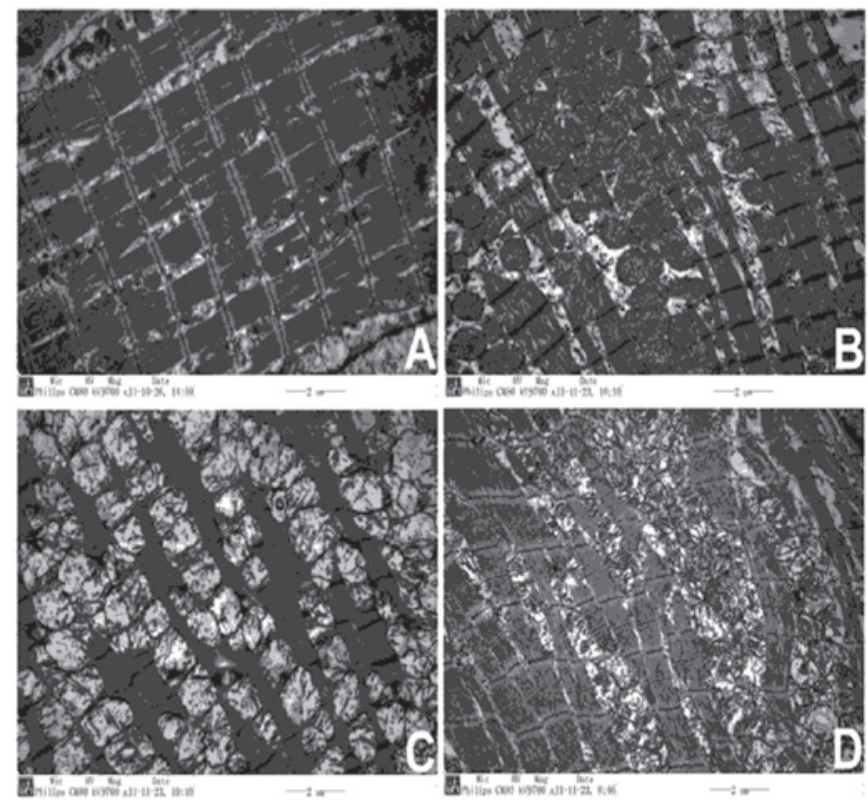

Figure 8. Pathological changes of the myocardium in experimental rats. At the end of animal study, heart tissue specimens were used for pathological examination using electron microscopy. (A) Wistar-Kyoto rat group; (B) RNA interference group; (C) negative group; (D) blank group. Magnification, $\mathrm{x} 9,700$.

experimental rats were compared. Among them, ratios of HW/BW and LVW/BW in the GPE-AGT-shRNA group $(2.92 \pm 0.15$ and $2.62 \pm 0.13 \mathrm{mg} / \mathrm{g}$ respectively) were significantly lower than those in the SHR blank controls $(3.64 \pm 0.22$ and $3.12 \pm 0.12 \mathrm{mg} / \mathrm{g}$; both $\mathrm{P}<0.05)$ or negative controls $(3.56 \pm 0.11$ and $3.2 \pm 0.09 \mathrm{mg} / \mathrm{g}$; both $\mathrm{P}<0.05)$, but marginally higher than those in the WKY group $(2.72 \pm 0.13$ and $2.48 \pm 0.21 \mathrm{mg} / \mathrm{g}$; both $\mathrm{P}>0.05$ ). 
Pathological changes of myocardial structure by microscopic examination. Pathological changes in myocardial structure in experimental rats were observed under an optical microscope. As shown in Fig. 7, hypertrophy of cardiac muscle cells was found in the blank and negative control SHR groups, but a significant improvement of cardiac hypertrophy was observed in the GPE-AGT-shRNA group.

Pathological changes of myocardial ultrastructure. Pathological changes of myocardial ultrastructure in experimental rats were observed under an electron microscope. Compared to the SHR control groups, integrity of the myocardial cell membrane as well as clear myofibrillar structures with tidy and defined cross striations were observed in the GPE-AGT-shRNA group. In this group, mitochondria without swelling and a small amount of local increase in myocardial interstitial collagen fibers with no obvious hyperplasia were observed, suggesting that GPE-AGT shRNA nanoparticles improved the myocardial ultrastructure in SHR.

\section{Discussion}

Hypertension is a lifelong disease, which is not only a cause of premature death and disability, but also a threat to social and economic development. It is estimated that, worldwide, the number of patients with hypertension will reach 1.5 billion in 2025, but only one-third of patients may be successfully treated (38). Reasons for the low control rate of hypertension include the short half-life of antihypertensive drugs, as well as their short duration of action, non-specific effects and side effects. Furthermore, poor compliance of hypertensive patients often occurs in clinical practice. Therefore, a therapy with long-term effects and reduced side effects is desirable for a lifelong treatment of hypertension.

RNAi technology is a recent breakthrough in post-transcriptional gene silencing. With this novel technique, expression of specific genes in mammalian cells can be inhibited (39-44). The formation of RNAi depends on the enzyme Dicer, which cleaves long double-stranded RNA (dsRNA) molecules into short double-stranded fragments of 20 nucleotides which are called siRNAs. siRNA is capable of blocking the mRNA expression of a specific gene, resulting in inhibition of subsequent protein expression, but avoiding non-specific degradation of genes and long double strand RNA-mediated cell death. Compared with technology of gene knockout, RNAi has the advantages of a short turnover time and low cost. Relative to the anti-sense nucleotide technique, RNAi has high efficiency, strong specificity and long-lasting duration. Therefore, RNAi has been widely applied in functional studies of specific genes in mammals (45), including cancer gene therapy $(46,47)$, viral infections (48-51) and monogenetic disorders (52).

The present study reported that injection of GPE-AGT-shRNA nanoparticles was able to significantly reduce the hepatic AGT mRNA expression, plasma levels of AGT and systolic blood pressure in SHR. In a control group, injection of GPE shRNA nanoparticles did not produce any similar effects. In the present study, no obvious side effects were present within 10 days following injection of nanoparticles. The intravenous injection of GPE-AGT-shRNA nanoparticles resulted in a significant decrease in blood pressure from days 1-7 after injection, as compared to the other treatments $(\mathrm{P}<0.05)$. The anti-hypertensive effect induced by GPE-AGT-shRNA nanoparticles lasted $>10$ days after a single injection. Administration of GPE-AGT-shRNA nanoparticles in SHR also had favorable effects on hypertension-induced cardiac hypertrophy. The reduction in blood pressure in SHR was $\sim 30 \mathrm{mmHg}$; however, the blood pressure did not normalize. Therefore, it may be assumed that the downregulation of hypertension in SHR was affected not only by AGT silencing, but also by other diverse factors in vivo. In addition, the results of the present study indicated that circulating AGT also has an important role in the pathogenesis of hypertension of SHR. Furthermore, the results showed that the reduction of circulating AGT following RNAi led to a decrease in hypertension in the SHR model. These findings are consistent with a previous study on AGT-knockout mice (53) or a study using anti-sense oligodeoxynucleotides (13). Due to the short period of time for observation of only 10 days after the injection in the present study, the accuracy of the results may have been affected. Therefore, to elucidate the exact effect of AGT on the development of hypertension and hypertrophy, further long-term studies are warranted.

In conclusion, intravenous injection with shRNA targeting the AGT gene coupled to the hepatic-specific GPE carrier decreased the plasma levels of AGT. The present study demonstrated that injection of AGT shRNA nanoparticles attenuated blood pressure, accompanied by improvement of the hypertension-induced cardiac hypertrophy in SHR. These findings suggested that RNAi may provide a potential therapeutic strategy for hypertension with cardiac hypertrophy.

\section{Acknowledgements}

This study was supported by grants from three projects, including the major scientific and technological specialized project sponsored by the National Natural Science Foundation of China (no. 81001416); the Shanghai Science and Technology Committee, China (no. 10JC1408902); the Research Fund for Integrated Medicine and Engineering of Shanghai Jiao Tong University (no. YG2011MS21); and the College subject of Shanghai Ninth People's Hospital Affiliated to Shanghai Jiao Tong University School of Medicine (no. 2012A13). The authors would also like to thank the Shanghai Key Laboratory of Tissue Engineering (Shanghai, China).

\section{References}

1. Wang Z, Hao G, Zhang L, Chen Z, Wang X, Guo M, Tian Y, Shao L and Zhu M: Central systolic blood pressure is associated with ethnicity and cardiovascular disease risk factors in Chinese middle-aged population. Eur J Prev Cardiol: Mar 27, 2015 (Epub ahead of print).

2. Cano-Gutierrez C, Reyes-Ortiz CA, Samper-Ternent R, Gélvez-Rueda JS and Borda MG. Prevalence and factors associated to hypertension among older adults in Bogotá, Colombia. J Aging Health: Mar 24, 2015 (Epub ahead of print).

3. Mahn JJ, Dubey E, Brody A, Welch R, Zalenski R, Flack JM, Ference B and Levy PD: Test characteristics of electrocardiography for detection of left ventricular hypertrophy in asymptomatic emergency department patients with hypertension. Acad Emerg Med 21: 996-1002, 2014.

4. Luft FC. Present status of genetic mechanisms in hypertension. Med Clin North Am 88: 1-18, 2004. 
5. Kern G, Mair SM, Noppert SJ, Jennings P, Schramek H, Rudnicki M, Mueller GA, Mayer G and Koppelstaetter C: Tacrolimus increases Nox4 expression in human renal fibroblasts and induces fibrosis-related genes by aberrant TGF-beta receptor signalling. PLoS One 9: e96377, 2014.

6. Pandey VG, Jain S, Rana A, Puri N, Arudra SK, Mopidevi B, Kaw M, Nasjletti A and Kumar A: Dexamethasone promotes hypertension by allele-specific regulation of the human angiotensinogen gene. J Biol Chem 290: 5749-5758, 2015.

7. Qi Y, Zhang K, Wu Y, Xu Z, Yong QC, Kumar R, Baker KM, Zhu Q, Chen S and Guo S: Novel mechanism of blood pressure regulation by forkhead box class O1-mediated transcriptional control of hepatic angiotensinogen. Hypertension 64: 1131-1140, 2014.

8. Alves M, Souza e Silva NA, Salis LH, Pereira Bde B, Godoy PH, Nascimento EM and Oliveira JM: Survival and predictive factors of lethality in hemodialysis: D/I polymorphism of the angiotensin I-converting enzyme and of the angiotensinogen M235T genes. Arq Bras Cardiol 103: 209-219, 2014.

9. Zhu X, Chang YP, Yan D, Weder A, Cooper R, Luke A, Kan D and Chakravarti A: Associations between hypertension and genes in the renin-angiotensin system. Hypertension 41: 1027-1034, 2003.

10. Kolder IC, Michels M, Christiaans I, Ten Cate FJ, Majoor-Krakauer D, Danser AH, Lekanne Deprez RH, Tanck M, Wilde AA, Bezzina CR, et al: The role of renin-angiotensin-aldosterone system polymorphisms in phenotypic expression of MYBPC3-related hypertrophic cardiomyopathy. Eur J Hum Genet 20: 1071-1077, 2012

11. Xu P, Wang Y, Sterner-Kock A, Bader M, Schultheiss HP and Walther T: Excessive hypertension and end-organ damage in a transgenic mouse line carrying the rat angiotensinogen gene. J Cardiovasc Pharmacol 53: 38-43, 2009.

12. Makino N, Sugano M, Ohtsuka S and Sawada S: Intravenous injection with antisense oligodeoxynucleotides against angiotensinogen decreases blood pressure in spontaneously hypertensive rats. Hypertension 31: 1166-1170, 1998.

13. Caulfield M, Lavender P, Farrall M, Munroe P, Lawson M, Turner P and Clark AJ: Linkage of the angiotensinogen gene to essential hypertension. N Engl J Med 330: 1629-1633, 1994.

14. Feng PF, Liu Y and Qin NP: Effect of sanwu hypotensive decoction on blood pressure and lymphokine activated killer cell in patient of primary hypertension and spontaneously hypotensive rats Zhong guo Zhong Xi Yi Jie He Za Zhi 21: 342-345, 2001.

15. Pinto YM, Paul M and Ganten D: Lessons from rat models of hypertension: From Goldblatt to genetic engineering. Cardiovasc Res 39: 77-88, 1998.

16. Wright JW, Mizutani S, Murray CE, Amir HZ and Harding JW Aminopeptidase-induced elevations and reductions in blood pressure in the spontaneously hypertensive rat. J Hypertens 8 : 969-974, 1990

17. Lemmer B, Mattes A, Böhm M and Ganten D: Circadian blood pressure variation in transgenic hypertensive rats. Hypertension 22 97-101, 1993

18. Lee RM: Structural alterations of blood vessels in hypertensive rats. Can J Physiol Pharmacol 65: 1528-1535, 1987.

19. Rippe B, Lundin S and Folkow B: Plasma volume, blood volume and transcapillary escape rate (TER) of albumin in young spontaneously hypertensive rats (SHR) as compared with normotensive controls (NCR). Clin Exp Hypertens 1: 39-50, 1978

20. Andresen MC, Krauhs JM and Brown AM: Relationship of aortic wall and baroreceptor properties during development in normotensive and spontaneously hypertensive rats. Circ Res. 43: 728-738, 1978.

21. Xi-Ye Fang (ed): Medical Experimental Zoology. 1st edition. People's Medical Publishing House, Beijing, p121, 1995.

22. Wakui H, Tamura K, Masuda S, Tsurumi-Ikeya Y, Fujita M, Maeda A, Ohsawa M, Azushima K, Uneda K, Matsuda M, et al: Enhanced angiotensin receptor-associated protein in renal tubule suppresses angiotensin-dependent hypertension. Hypertension 61 : 1203-1210, 2013

23. Nabeshima Y, Tazuma S, Kanno K, Hyogo H and Chayama K Deletion of angiotensin II type I receptor reduces hepatic steatosis. J Hepatol 50: 1226-1235, 2009.

24. Yue H, Li W, Desnoyer R and Karnik SS: Role of nuclear unphosphorylated STAT3 in angiotensin II type 1 receptor-induced cardiac hypertrophy. Cardiovasc Res 85: 90-99, 2010.

25. Chen X, Qiu Z, Yang S, Ding D, Chen F, Zhou Y, Wang M, Lin J, $\mathrm{Yu} \mathrm{X}$, Zhou Z, et al: Effectiveness and safety of a therapeutic vaccine against angiotensin II receptor type 1 in hypertensive animals. Hypertension 61: 408-416, 2013.
26. He J, Bian Y, Gao F, Li M, Qiu L, Wu W, Zhou H, Liu G and Xiao C: RNA interference targeting the ACE gene reduced blood pressure and improved myocardial remodelling in SHRs. Clin Sci (Lond) 116: 249-255, 2009.

27. Lu P, Yuan L, Wang Y, Du Q and Sheng J. Effect of GPE-AGT nanoparticle shRNA transfection system mediated RNAi on early atherosclerotic lesion. Int J Clin Exp Pathol 5: 698-706, 2012.

28. Gong H, Liu CM, Liu DP and Liang CC: The role of small RNAs in human diseases: Potential troublemaker and therapeutic tools. Med Res Rev 25: 361-381, 2005.

29. Park TG, Jeong JH and Kim SW: Current status of polymeric gene delivery systems. Adv Drug Deliv Rev 58: 467-486, 2006.

30. Srinivas R, Samanta S and Chaudhuri A: Cationic amphiphiles: Promising carriers of genetic materials in gene therapy. Chem Soc Rev 38: 3326-3338, 2009.

31. Mintzer MA and Simanek EE: Nonviral vectors for gene delivery. Chem Rev 109: 259-302, 2009.

32. Yu H and Wagner E: Bioresponsive polymers for nonviral gene delivery. Curr Opin Mol Ther 11: 165-178, 2009.

33. Wang YQ, Su J, Wu F, Lu P, Yuan LF, Yuan WE, Sheng J and Jin T: Biscarbamate cross-linked polyethylenimine derivative with low molecular weight, low cytotoxicity, and high efficiency for gene delivery. Int J Nanomedicine 7: 693-704, 2012.

34. Lu P, Yuan L, Wang Y, Du Q and Sheng J: Effect of GPE-AGT nanoparticle shRNA transfection system mediated RNAi on early atherosclerotic lesion. Int J Clin Exp Pathol 5: 698-706, 2012.

35. Wang Y, Su J, Cai W, Lu P, Yuan L, Jin T, Chen S and Sheng J: Hepatocyte-targeting gene transfer mediated by galactosylated poly(ethylene glycol)-graft-polyethylenimine derivative. Drug Des Devel Ther 7: 211-221, 2013.

36. Zhang X, Gao Y, Dong J, Wang S, Yao B, Zhang J, Hu S, Xu X, Zuo H, Wang L, et al: The compound Chinese medicine 'Kang Fu Ling' protects against high power microwave-induced myocardial injury. PLoS One 9: e101532, 2014

37. Silvani A, Bastianini S, Berteotti C, Cenacchi G, Leone O, Lo Martire V, Papa V and Zoccoli G: Sleep and cardiovascular phenotype in middle-aged hypocretin-deficient narcoleptic mice. J Sleep Res 23: 98-106, 2014.

38. Li D, Lv J, Liu F, Liu P, Yang X, Feng Y, Chen G and Hao M: Hypertension burden and control in mainland China: Analysis of nationwide data 2003-2012. Int J Cardiol 184: 637-644, 2015.

39. Khandelia P, Yap K and Makeyev EV: Streamlined platform for short hairpin RNA interference and transgenesis in cultured mammalian cells. Proc Natl Acad Sci USA 108: 12799-12804, 2011

40. Maillard PV, Ciaudo C, Marchais A, Li Y, Jay F, Ding SW and Voinnet O: Antiviral RNA interference in mammalian cells. Science 342: 235-238, 2013.

41. Li Y, Lu J, Han Y, Fan X and Ding SW: RNA interference functions as an antiviral immunity mechanism in mammals. Science 342 : 231-234, 2013

42. Lebedev TD, Spirin PV and Prassolov VS: Transfer and expression of small interfering RNAs in mammalian cells using lentiviral vectors. Acta Naturae 5: 7-18, 2013.

43. Hannon GJ: RNA interference. Nature 418: 244-251, 2002.

44. Pare JM, LaPointe P and Hobman TC: Hsp90 cochaperones p23 and FKBP4 physically interact with hAgo2 and activate RNA interference-mediated silencing in mammalian cells. Mol Biol Cell 24: 2303-2310, 2013.

45. Paddison PJ: RNA interference in mammalian cell systems. Curr Top Microbiol Immunol 320: 1-19, 2008.

46. Dalmay T: MicroRNAs and cancer. J Intern Med 263: 366-375, 2008.

47. Ohkumo T, Masutani C, Eki T and Hanaoka F: Use of RNAi in C. elegans. Methods Mol Biol 442: 129-137, 2008.

48. Huang DD: The potential of RNA interference-based therapies for viral infections. Curr HIV/AIDS Rep 5: 33-39, 2008.

49. Chen Y, Cheng G and Mahato RI: RNAi for treating hepatitis B viral infection. Pharm Res 25: 72-86, 2008

50. Volarevic M, Smolic R, Wu CH and Wu GY: Potential role of RNAi in the treatment of HCV infection. Expert Rev Anti Infect Ther 5: 823-831, 2007.

51. Singh SK: RNA interference and its therapeutic potential against HIV infection. Expert Opin Biol Ther 8: 449-461, 2008.

52. Ying SY and Lin SL: Current perspectives in intronic micro RNAs (miRNAs). J Biomed Sci 13: 5-15, 2006

53. Chen D, Hazelwood L, Walker LL, Oldfield BJ, McKinley MJ and Allen AM: Changes in angiotensin type 1 receptor binding and angiotensin-induced pressor responses in the rostral ventrolatera medulla of angiotensinogen knockout mice. Am J Physiol Regul Integr Comp Physiol 298: R411-R418, 2010. 